\title{
Adalimumab efficacy in Bulgarian patients with Crohn's disease in a single study center
}

\section{Luben Milatchkov', Dobriana Panova1, Zoia Spassova', Rosen Nikolov ${ }^{1}$}

1. University Hospital "St. Ivan Rilski", Clinic of Gastroenterology, Medical University - Sofia, Bulgaria

\begin{abstract}
Adalimumab, an antitumor necrosis factor monoclonal antibody, is effective in treating moderate-toseverely active Crohn's disease. The purpose of our study is to determine clinical and endoscopic response during one-year Adalimumab therapy in Crohn's disease. Materials and methods: We have included 17 patients with moderately active, azathioprine resistant Crohn's disease; 9 of these patients had stenosis and 6 had fistulas. All of the patients were treated with Adalimumab. All of them were followed-up for 12 months. For evaluation of response to therapy we have used Crohn's Disease Activity Index, C-reactive protein and hemoglobin. Results: At week 12 fourteen patients experienced clinical improvement (reduction in Crohn's Disease Activity Index >100 points); all of the fistulas stopped draining. Significant improvement of anemia was observed after 12 months of Adalimumab therapy ( $p=0.027$ ), as well as complete endoscopic remission. Of all patients that were treated for 12 months, 3 experienced endoscopic relapse. Neither closure of fistulas was observed, nor were stenosing aeries improved during Adalimumab therapy. Conclusion: Achievement of endoscopic remission with biological therapy also leads to improvement in anemia. After one-year biological therapy endoscopic relapse can be observed. One year of Adalimumab therapy has diminished fistula draining but no closure was observed.
\end{abstract}

Keywords: Adalimumab, Crohn's disease, Crohn's Disease Activity Index, endoscopy, remission 


\section{Background}

Adalimumab (Humira) is a subcutaneously (SC) self-administered recombinant fully-human monoclonal immunoglobulin (IgG1) antibody. It binds with a high affinity and specificity to soluble tumor necrosis factor (TNF $\alpha$ ) and neutralizes its biological function by blocking its interaction with TNF receptors (1). Given the central role of TNF in the inflammatory cascade, Adalimumab has potential benefit in a plethora of inflammatory and immune-mediated disorders. The SC route of administration offers patients the additional benefit of convenience since medication administration can be done without travelling to an infusion center thus offering patients the opportunity to avoid missing time off from work or schooling for infusion (when compared to Infliximab).

Infliximab was the first TNF $\alpha$ inhibitor approved for the treatment of Crohn's disease (CD) in the US. Similar to Infliximab, Adalimumab has been evaluated for its effect in inducing and maintaining remission, its steroid-sparing effect, and its impact on hospitalizations and surgeries.

The aim of our study is to determine the long term clinical and endoscopic response to Adalimumab (Humira, AbbVie) therapy in patients with CD in a Bulgarian single study center.

\section{Materials and Methods}

We carried out a descriptive, observational study that included 17 patients with a mean age of 36 years with moderately active, Azathioprine (AZA) resistant CD, diagnosed and followed-up between January 2013 and January 2014 in the Clinic of Gastroenterology of St. Ivan Rilski Hospital in Sofia, Bulgaria. All patients were naïve to anti-TNF $\alpha$. Exclusion criteria were the presence of active infection; heart, renal or liver failure and neurological or immunodeficiency disorders. Infection with Tuberculosis was excluded using Mantoux and Quantiferon-TB-Gold test prior to starting treatment. 9 of the CD patients had stenosis and 6 had fistulas. All of the evaluated patients were treated with ADA according to the accepted criteria of ECCO and the Bulgarian consensus for Inflammatory Bowel disease (IBD). All of the patients were followed-up for 12 months.

\section{Patients' characteristics}

Table 1. Demographic and clinical data for patients with CD treated with ADA.

\begin{tabular}{|c|c|}
\hline Total number of patients & $\mathbf{n}=\mathbf{1 7}$ \\
\hline Characteristics & Data \\
\hline Age & $36(18-61)$ years \\
\hline Sex & \\
Female & 10 \\
Male & 7 \\
\hline Age at onset & 2 \\
A1 (<16 years) & 13 \\
\hline
\end{tabular}




\begin{tabular}{|c|c|}
\hline A2 (17-40 years) & 2 \\
A3 (>40 years) & \\
\hline Location & \\
L1 (ileal) & 0 \\
L2 (colonic) & 7 \\
L3 (ileocolonic) & 10 \\
L4 (isolated upper disease) & 0 \\
\hline Behaviour * & 5 \\
B1(non- stricturing, non- penetrating) & 9 \\
B2 (stricturing) & 6 \\
B3 (penetrating) & 5 \\
Perianal disease & 13 \\
\hline Therapy & 14 \\
\hline Immunomodulators (AZA, MP) & \\
\hline Corticosteroids & \\
\hline
\end{tabular}

* some patients have both stricturing and penetrating forms of CD disease.

For disease's activity assessment we applied Crohn's Disease Activity Index (CDAI) and C-reactive protein (CRP). Additionally we determined and followed-up during therapy the levels of haemoglobin $(\mathrm{Hb})$, albumin, cholesterol (CHO), triglycerides (TGs) and endoscopic activity.

We present CD patients according to the presence of anaemia or not, as well as, to the presence of high $\mathrm{CRP}$ levels and CRP levels in referent range in the tables below.

Table 2. CD patients with anaemia before ADA therapy according to the referent ranges of haemoglobin $(\mathrm{Hb})$ of WHO.

\begin{tabular}{|c|c|}
\hline Number of patients & $\mathbf{H b} \mathbf{g} / \mathbf{l}$ level \\
\hline 1 & 91 \\
\hline 1 & 107 \\
\hline 2 & 115 \\
\hline 1 & 119 \\
\hline
\end{tabular}

*Haemoglobin mean value $108 \mathrm{~g} / \mathrm{l}$ (91-119) and median value $111 \mathrm{~g} / \mathrm{l}$ in CD patients with anaemia.

Table 3. $\mathrm{CD}$ patients with $\mathrm{Hb}$ values in referent range before ADA therapy according to WHO. 


\begin{tabular}{|c|c|}
\hline Number of patients & $\mathbf{H b} \mathbf{g} / \mathbf{l}$ \\
\hline 1 & 121 \\
\hline 1 & 123 \\
\hline 1 & 129 \\
\hline 2 & 131 \\
\hline 2 & 132 \\
\hline 2 & 152 \\
\hline 2 & 154 \\
\hline 1 & 174 \\
\hline
\end{tabular}

Before ADA therapy, twelve of the CD patients had haemoglobin levels from $121 \mathrm{~g} / \mathrm{l}$ to $174 \mathrm{~g} / \mathrm{l}$. The mean value of haemoglobin was $140 \mathrm{~g} / \mathrm{l}$ while the median value $-132 \mathrm{~g} / \mathrm{l}$

Table 4. Normal and elevated CRP in patients with CD before ADA therapy.

\begin{tabular}{|l|l|l|}
\hline CRP level & CRP $<6(\mathrm{mg} / \mathrm{l})$ & CRP $>6(\mathrm{mg} / \mathrm{l})$ \\
\hline Characteristics & \multicolumn{2}{|l|}{} \\
\hline Number of patients & 5 & 12 \\
\hline Mean $(\mathrm{mg} / \mathrm{l})$ & 1,4 & 23,5 \\
\hline Median $(\mathrm{mg} / \mathrm{l})$ & 1 & 18 \\
\hline Min $(\mathrm{mg} / \mathrm{l})$ & 1 & 8 \\
\hline Max $(\mathrm{mg} / \mathrm{l})$ & 2 & 44 \\
\hline
\end{tabular}

Five of the CD patients had normal CRP range towards twelve $C D$ patients with $C R P>6$. From $C D$ patients with high CRP, the mean CRP value was 23,54.

\section{Results}

\section{Efficacy evaluation}

In CD patients with anaemia, Hb levels significantly increased at week $12(p=0.002)$, at week $24(p=$ $0.020)$ and at week $48(p=0.002)$ of ADA therapy (Figure 1). 
Figure 1. Hemoglobin levels in CD patients with anemia over 48 weeks of treatment with Adalimumab.

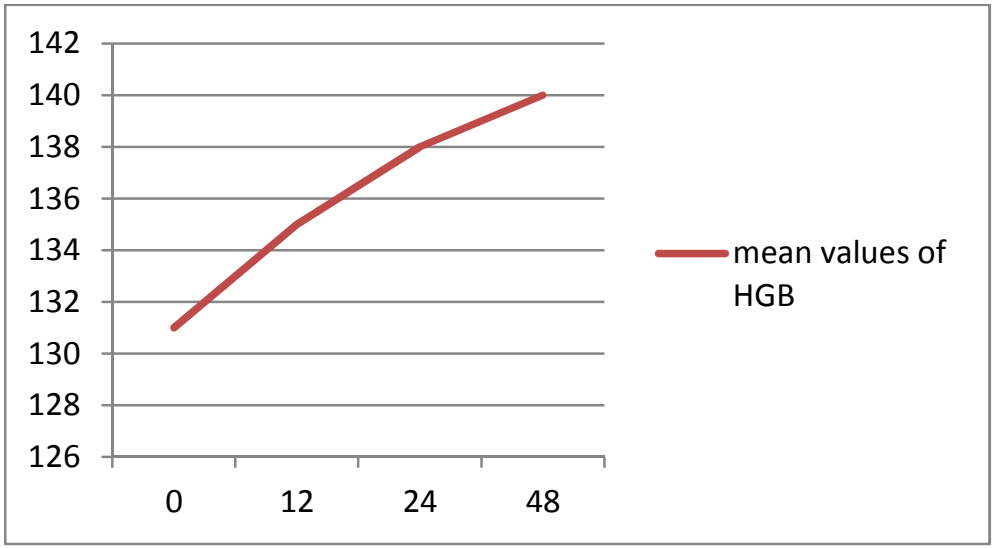

At week 12 , only one patient is registered with anaemia, while at week 24 , none of the patients have $\mathrm{Hb}$ under reference ranges of $\mathrm{WHO}$.

Before starting therapy with ADA, 5 patients had CRP $<6 \mathrm{mg} / \mathrm{l}$ against 12 patients with CRP $>6 \mathrm{mg} / \mathrm{l}$.

Analysing the dynamics of CRP mean values in patients with $\mathrm{CRP}>6 \mathrm{mg} / \mathrm{l}$, we can notice a significant CRP reduction at week $12(p=0,019)$. This trend continued until week $24(p=0,117)$ (Figure 2$)$.

Figure 2. Dynamics of mean values of CRP $\mathrm{mg} / \mathrm{l}$

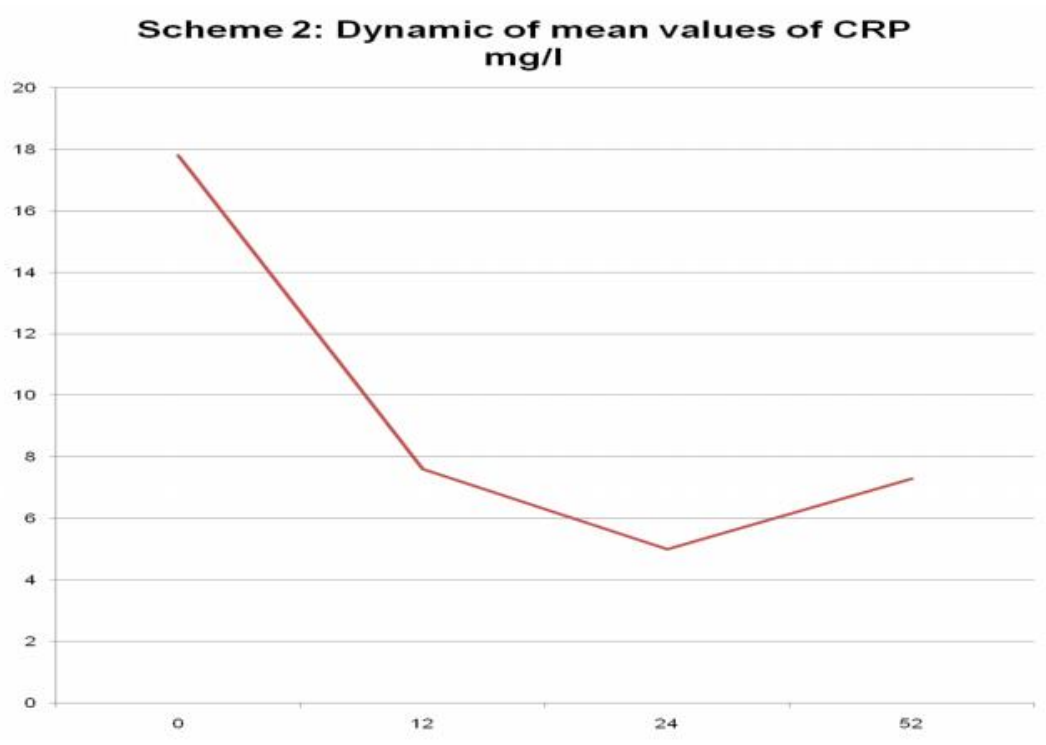


On the other hand, although there is a slight increase $(p=0,083)$ of CRP at week 52 in comparison with week 24, CRP level remains under $7 \mathrm{mg} / \mathrm{l}$.

Only 2 patients were registered with hypoalbuminaemia before ADA therapy. At week 12 all patients had normal albumin level.

We also observed the initial levels of TGs that were $1 \pm 0.5(0.5-3.0) \mathrm{mmol} / \mathrm{l}$. After treatment with Adalimumab the levels increased to $1.5 \pm 1.5(0.4-7) \mathrm{mmol} / \mathrm{l}$ but these changes were not significant $(\mathrm{p}=$ 0.11). Even though insignificant, before treatment there was only one patient with abnormal TGs while after Adalimumab injections, we observed six people (35\%) with elevated TGs.

The initial level of $\mathrm{CHO}$ was $4.4 \pm 1.2(2.4-6.9) \mathrm{mmol} / \mathrm{l}$. After treatment with Adalimumab no change was registered - 4,5 $\pm 1,3(2.5-7.7) \mathrm{mmol} / \mathrm{l},(\mathrm{p}=0.06)$.

At the beginning of the therapy 8 patients (47\%) had liver steatosis, while, during anti-TNF-treatment 10 patients $(59 \%)$ had fatty liver, which is again an insignificant change $(p=0.43)$.

\section{Response to treatment}

According to CDAl score, 14 patients treated with ADA experienced clinical remission at week 24 (Figure $3)$.

Figure 3. Dynamics of mean values of CDAl score during therapy with ADA

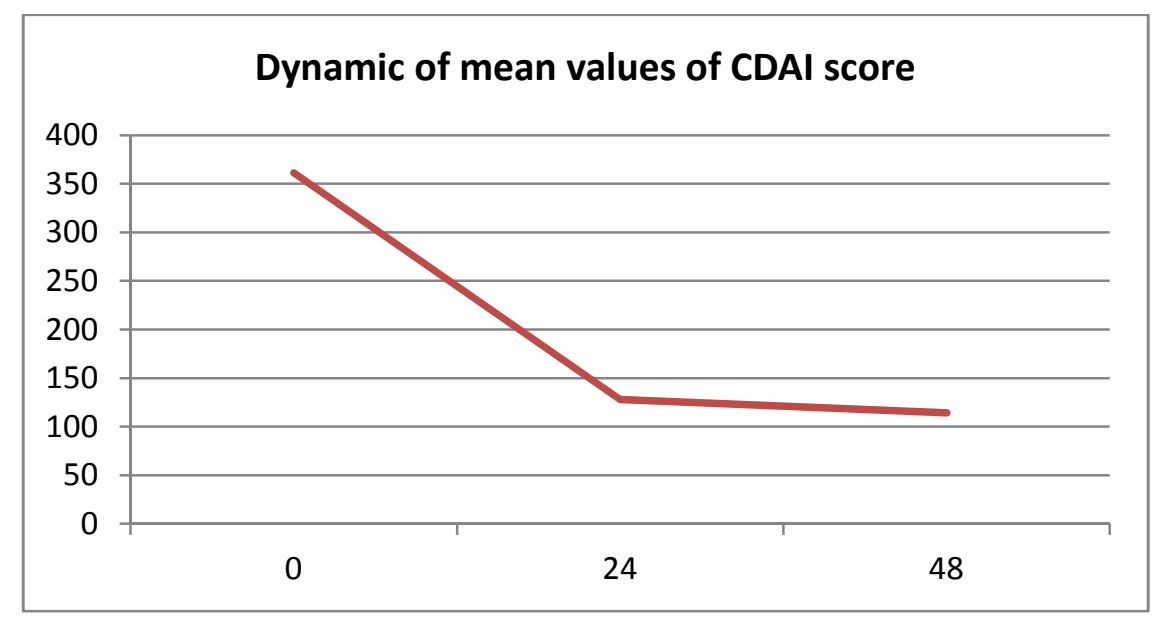

Of the 17 patients administered ADA, 3 failed to register a response. In these patients the results of faecal Calprotectin test were elevated $>1000 \mathrm{mg} / \mathrm{kg}$. Patients who did not show a response to therapy required intensification of the regimen to $40 \mathrm{mg}$ ADA SC per week.

In the period under study no one experienced any surgical procedure. 
All of the fistulas stopped draining. In two of the patients a new fistula was registered. Neither closure of fistulas was observed, nor were stenosing aeries improved during ADA therapy.

\section{Safety evaluations}

Infusion and injection site reactions were not observed. Serious adverse events were not reported. No malignant neoplasms, no opportunistic infections and no tuberculosis (TB) were registered.

\section{Discussion}

Over recent years, the management of IBD has dramatically changed. In particular, advances in understanding the pathogenesis and the natural course of the disease have substantially changed the therapeutic algorithms with the introduction of new biological drugs. Among these the anti-TNF- $\alpha$ monoclonal antibodies Infliximab and Adalimumab are currently being approved for the management of $C D$, in particular for patients with moderately and severely active luminal disease who are unresponsive to conventional therapy.

In our study, we have demonstrated that one year of Adalimumab treatment mainly decreases the number of liquid and soft stools, improves the abdominal pain, the general well-being of the patient and decreases the frequency of complications. The reduction of CDAI score influences and improves the patient's quality of life; improves daily activities, reduces absence from work and achieves improved patient's social inclusion and mental health.

Although CD patients experienced anaemia improvement in our study, no mucosal healing was observed endoscopically during one year treatment with ADA.

Different studies including ADA treatment in IBD show that mucosal improvement in CD occurs more often and faster in patients with involvement of a small area of the mucosa.

Results from the clinical trial EXTEND demonstrated that Adalimumab (ADA)-treated patients (pts) with moderately to severely active Crohn's disease (CD) were more likely to achieve mucosal healing (MH) than pts receiving placebo (PBO) (2).

Moreover, longer term treatment with ADA is needed ( $>1$ year) to expect mucosal healing.

After prolonged biological therapy intestinal absorption improves, patients broaden their diet, gain weight and serum lipid levels might be influenced. In consequence, some patients develop fatty liver. Another explanation of the increase in serum lipid levels (triglycerides, cholesterol) and ultrasound data of hepatic steatosis may be an ADA's side effect. Further investigation is needed in this direction. A meta-analysis between January 2004 and October 2012 covering adverse side effects reports of Adalimumab state that 10 individuals taking ADA reported hepatic steatosis to the FDA (3).

ADA therapy decreased fistula draining but no closure was observed. 
Faecal Calprotectin is an excellent inflammatory marker and its levels have been found to correlate with the severity of colon involvement and response to therapy with ADA in patients with CD.

When weighing the risks and benefits of biologic therapy for patients with IBD, physicians must account for the consequences of undertreated IBD. These include the direct costs of hospitalizations and operations for IBD, the direct costs of treatment for side effects associated with chronic, nonbiologic therapies, and indirect costs associated with lost productivity or nonmonetary costs such as quality-of-life (QOL) decrements (5).

In summary, we have demonstrated that one-year ADA treatment is effective in CD patients who develop loss of response to immunosuppressive therapy with Azathioprine or are naïve to biological treatment.

This study's main limitation is the small sample size, essentially due to the fact that all patients were recruited from a single hospital. Multicenter longitudinal studies with longer follow-up periods and a greater number of analyzed patients are required to determine the true efficacy and safety of long-term ADA treatment.

\section{Conclusion and Clinical Significance}

Treatment with TNF $\alpha$ inhibitors has been a significant advance in the treatment of IBD. Adalimumab, a fully human, monoclonal antibody can be self-administered every week to every other week.

Achievement of clinical and endoscopic remission with biological therapy also leads to improvement in anaemia. After prolonged biological therapy intestinal absorption improves, patients broaden their diet and serum lipid levels might be influenced. ADA therapy diminishes fistula draining but no closure is observed. Adalimumab is well tolerated and no serious side-effects were noticed.

\section{References}

1. Sorbera LA, Rabasseda X, Castaner RM. Adalimumab. Drugs Future 2001;26:639-646.

2. Sandborn WJ, Wolf D. Colombel JF, et al. Adalimumab achieves efficacy in mucosal healing regardless of baseline disease severity in patients with Crohn's disease: Data from EXTEND P572.

3. MedsFacts Meta-Analysis covering adverse side effect reports of ADALIMUMAB patients who developed HEPATIC STEATOSIS.

4. Clark M, Colombel JF, Feagan BC, et al. American Gastroenterological Association consensus development conference on the use of biologics in the treatment of inflammatory bowel disease Gastroenterology 2006;133:312-339.

5. Rutgeerts $\mathrm{P}$, Diamond $\mathrm{RH}$, Bala $\mathrm{M}$, et al. Scheduled maintenance treatment with infliximab is superior to episodic treatment for the healing of mucosal ulceration associated with Crohn's disease. Gastrointestinal Endoscopy 2006;63:433-442. 


\section{Corresponding author:}

Luben Milatchkov

15 Akad. Ivan Geshov Blvd., Sofia 1431, Bulgaria

University Hospital St. Ivan Rilski

Clinic of Gastroenterology

E-mail:lub26@hotmail.com,

fax number: +35928510615 